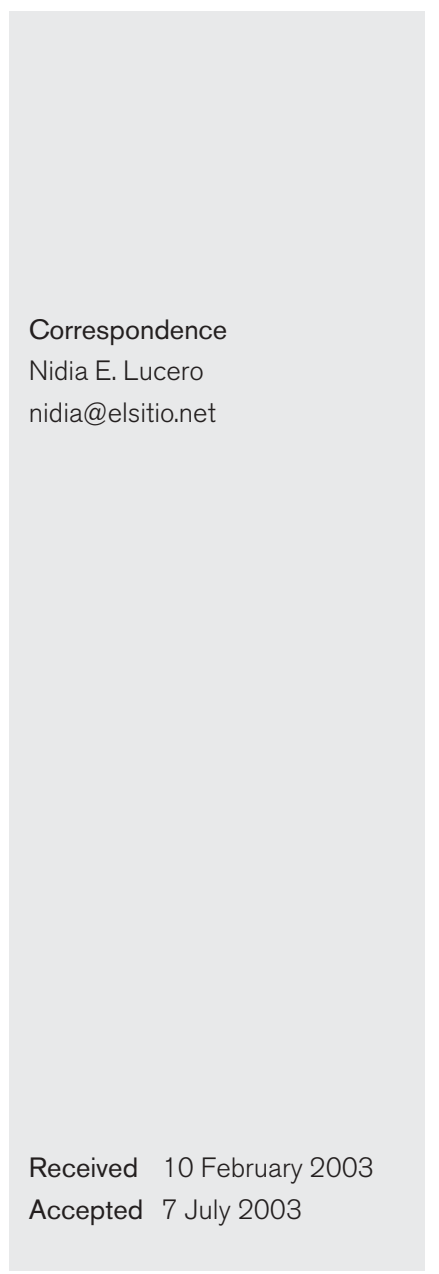

\title{
Fluorescence polarization assay for diagnosis of human brucellosis
}

\author{
Nidia E. Lucero, ${ }^{1}$ Gabriela I. Escobar, ${ }^{1}$ Sandra M. Ayala, ${ }^{1}$ Patricia Silva Paulo ${ }^{2}$ \\ and Klaus Nielsen ${ }^{3}$ \\ ${ }^{1}$ Brucellosis Laboratory, Administración Nacional de Laboratorios e Institutos de Salud 'Dr C. G. \\ Malbrán', Avda. Velez Sarsfield 563, 1281 Buenos Aires, Argentina \\ ${ }^{2}$ Comisión Nacional de Energia Atómica, Centro Atómico de Ezeiza, Buenos Aires, Argentina \\ ${ }^{3}$ Canadian Food Inspection Agency, Animal Diseases Research Institute, Nepean, Ontario, Canada \\ $\mathrm{K} 2 \mathrm{H} 8 \mathrm{P} 9$
}

Fluorescence polarization immunoassay (FPA) uses molecular rotational properties to measure antibody binding to antigen directly. The potential use of this method was assessed in comparison to a competitive enzyme immunoassay (CELISA) and conventional serological tests for the diagnosis of brucellosis on a total of 587 human sera. Based on 340 sera from asymptomatic blood donors with no evidence of brucellosis, the specificity of the FPA was $97.9 \%$ using a cut-off value of $72 \mathrm{mP}$. Sera from Brucella-infected patients (11 Brucella melitensis, 32 Brucella abortus, 32 Brucella suis and one Brucella sp.) yielded a sensitivity estimate of $96.1 \%$. In tests on 84 sera from suspected brucellosis patients, the FPA detected 80 cases. Of 87 sera from patients with probable infection, 15 were detected by both CELISA and FPA, three by CELISA only and four by FPA only. The discrepancies in both groups involved sera with low, declining titres. The FPA uses a sample of $40 \mu \mathrm{l}$ serum, takes about 5 min to complete and has been demonstrated to be accurate for the detection of antibodies to $B$. abortus, $B$. melitensis and $B$. suis and for identifying patients suffering relapses. Because of the ease of the procedure, it could be readily adopted for use in clinical laboratories and blood banks.

\section{INTRODUCTION}

Human brucellosis is diagnosed on the basis of clinical findings and laboratory studies that include bacteriological and serological tests. The conventional serological tests are insufficiently sensitive and/or specific to be used individually and, as a result, a panel of tests is frequently used, often leading to difficulty in interpreting the results. To overcome some of these problems, newer serological tests based on primary binding assays have been developed, mainly to improve test sensitivity and specificity. A competitive enzyme immunoassay (CELISA) for the detection of serum antibody to Brucella has been shown to be a suitable test for human brucellosis (Lucero et al., 1999). The CELISA uses a $\mathrm{mAb}$ specific for a common and repeating epitope on the polysaccharide portion of the smooth lipopolysaccharide molecule of Brucella (S-LPS) to compete with antibody in the sample. This results in an assay with higher specificity than other assays because it frequently eliminates cross-reactions with other antigens.

Abbreviations: CELISA, competitive ELISA; FPA, fluorescence polarization assay.
Fluorescence polarization immunoassay (FPA) makes use of molecular rotational properties, measuring antibody binding to antigen directly, eliminating the need for separation procedures. The principle of the method relies on a fluorescent dye attached to a small antigen (or antibody fragment) that is excited by plane-polarized light at the appropriate wavelength. The rate of rotation of the antigen molecule is reduced when its molecular size is increased by its binding to antibody (or antigen). This change in rate can be measured. The method has been applied to the detection of bovine antibody to Brucella abortus, resulting in a sensitive and specific test (Nielsen et al., 2000). The assay, which can be completed in a few minutes, requires a one-step serum dilution, assessment of background fluorescence, addition of labelled antigen and, finally, measurement of antigen-antibody interaction. In this study, we compare results obtained with the FPA with the CELISA and conventional tests for the diagnosis of human brucellosis.

\section{METHODS}

Serum samples. Serum samples were divided into the following groups: 340 sera from blood donors (18-62 years old) with no clinical or 
epidemiological evidence of brucellosis and negative plate agglutination test (PAT), Rose Bengal test (RB), buffered antigen plate agglutination test (BPA), standard tube agglutination test (TAT), cold complement fixation test $(\mathrm{CF})$ and CELISA; 76 sera from patients from whom Brucella sp. was isolated; 84 sera from suspected brucellosis patients with clinical symptoms compatible with brucellosis and positive PAT, RB, BPA, TAT, CF and CELISA at any titre and 87 sera from patients probably infected (slaughterhouse workers and blood donors) but free of clinical symptoms compatible with brucellosis yet positive for one or more of the serological tests at low titres.

Serological tests. PAT, RB, BPA, TAT and CF were performed as described previously (Centro Panamericano de Zoonosis, 1981; Angus \& Barton, 1984; Alton et al., 1988; Lucero \& Bolpe, 1998). The antigens used in the above tests were prepared at the Administración Nacional de Laboratorios e Institutos de Salud (ANLIS) 'Dr C. G. Malbrán', using antigens from the National Veterinary Services Laboratories, US Department of Agriculture, for reference purposes.

CELISA involved adsorption of Brucella S-LPS antigen to polystyrene plates and was performed as described previously (Nielsen et al., 1995, 1996; Lucero et al., 1999). Briefly, plates with antigen added were incubated overnight at $4{ }^{\circ} \mathrm{C}$ followed by the addition of mouse $\mathrm{mAb}$ specific for a common epitope of the S-LPS molecule and diluted test sera. After the incubation period, diluted goat anti-mouse IgG antibody conjugated with horseradish peroxidase was added, followed by the addition of substrate and chromogen.

If the test was positive, the serum competed with the $\mathrm{mAb}$ for the epitope sites and inhibited binding of the mAb to the S-LPS antigen and subsequent colour development. Results for control and test sera were expressed as percentage inhibition (PI) of mAb activity against the antigen. For interpretation, sera with PI values of $28 \%$ or more were considered positive. The S-LPS (B. abortus S 1119-3), mAb (M84) and control sera (strongly positive and weakly positive bovine anti-Brucella serum and negative bovine serum) were standardized and supplied by the Brucellosis Center of Expertise and OIE Reference Laboratory, Animal Diseases Research Institute (ADRI), Canada. In addition to bovine serum controls, positive and negative reference human sera were included in each CELISA plate. The conjugate, pre-adsorbed with bovine, equine and human serum protein, was from Jackson Laboratories.

FPA. FITC was conjugated to O-polysaccharide (OPS) extracted from S-LPS of B. abortus S 1119-3 and standardized at ADRI (Nielsen et al., 2000). For testing, control and test sera were diluted 1:50 in $2 \mathrm{ml} \mathrm{0.01 \textrm {M }}$ sodium phosphate, $\mathrm{pH} 7 \cdot 4$, containing $0 \cdot 15 \mathrm{M} \mathrm{NaCl}, 0 \cdot 1 \%$ sodium azide and $0.05 \%$ lithium dodecylsulfate (PBSAL), in a $12 \times 75 \mathrm{ml}$ glass tube. A serum blank measurement was obtained with a Fluorescence Polarization Analyser (FPM-1; Jolley Consulting and Research), with the lamp feedback off and the heater set at $37^{\circ} \mathrm{C}$. Twenty microlitres FITC-conjugated OPS was added and, after gentle mixing and incubation at room temperature for at least $2 \mathrm{~min}$, a second measurement was made with the FPM-1, which automatically subtracted the blank reading. Positive and negative reference human sera were included with each lot of 30 samples tested. Data, expressed as millipolarization units $(\mathrm{mP})$, were indicative of the amount of antibody present in the serum sample.

Bacteriological examination. Brucella organisms were isolated from three blood cultures incubated in $10 \% \mathrm{CO}_{2}$ using a liquid medium ( 1.5 g NZ amine, $0.5 \mathrm{~g}$ Primatone, $0.2 \mathrm{~g}$ yeast extract, $0.1 \mathrm{~g}$ glucose, $0.5 \mathrm{~g}$ $\mathrm{NaCl}, 0.01 \mathrm{~g}$ sodium bisulphite, $2.5 \mathrm{~g}$ sodium citrate, $100 \mathrm{ml}$ distilled water). A bottle holding $25 \mathrm{ml}$ liquid medium was inoculated with $5 \mathrm{ml}$ blood (Garcia-Carrillo \& Lucero, 1993). Cultures were kept for 45 days before being considered negative. A suspected Brucella culture was subcultured on solid medium for identification. The strains were typed as recommended by the former ICSB Subcommittee on the Taxonomy of the Genus Brucella (Corbel \& Brinley-Morgan, 1984) at ANLIS 'Dr C. G. Malbrán’.

Data analysis. An initial cut-off value was calculated from the FPA data obtained from the negative sera. Diagnostic sensitivity and specificity were calculated by plotting the data for negative and positive samples using a frequency histogram. In addition, the data were studied using receiver operator characteristic (ROC) analysis, which calculates the most suitable cut-off value, estimated by comparing the range of sensitivity and specificity values (Metz, 1978; Schoonjans et al., 1995).

\section{RESULTS AND DISCUSSION}

The 340 negative sera yielded a mean FPA value of $60.95 \mathrm{mP}$ with a standard deviation of $5 \cdot 28 \mathrm{mP}$. A frequency distribution showed a characteristic skewing of the results for the serum samples (data not shown). The cut-off values were confirmed by ROC analysis (Fig. 1) using 76 sera of Brucellainfected patients as positive sera (defined on the basis of positive clinical history, cultural results and reactions with TAT, CF and CELISA). Thus, if a cut-off value of $72 \mathrm{mP}$ was selected (mean $+2 \mathrm{SD}$ ), the sensitivity and specificity values were respectively determined to be $96 \cdot 1$ and $97.9 \%$ (seven false-positives and three false-negative results were evident; Table 1).

Seventy-six isolates of Brucella were obtained from patients. Of these, typing results yielded 11 isolates of Brucella melitensis [biovar 1 or 1a (atypical strains; data not shown)], 32 B. abortus (biovar 1, except for three isolates of biovar 2 and one of S19), 32 Brucella suis (biovar 1 or 1a; Corbel et al., 1984) and one isolate that was not typed. In general, the results of serological tests used were consistent, but some discrepancies were found (Table 2). The FPA detected 73 culture-positive cases (ranging from 73 to $255 \mathrm{mP}$ ), CELISA 74, CF 73 and TAT 73 (but three sera had titres $<1: 100$ ).

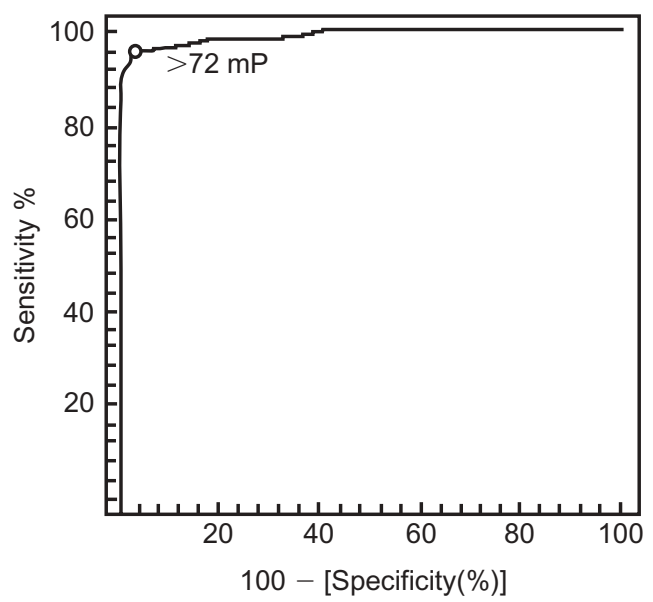

Fig. 1. ROC analysis of sensitivity (\%) plotted against 100-specificity (\%) for various cut-off values of the FPA for detection of antibody to Brucella sp. A value of $72 \mathrm{mP}$ gave the maximum sum of sensitivity and specificity and was considered to be the optimum cutoff value. 
Table 1. Sensitivity and specificity determinations for FPA data

FPA results are compared with culture results for the positive population and absence of clinical/epidemiological/serological evidence of brucellosis for the negative population. A cut-off value of $72 \mathrm{mP}$ was used for the FPA. Sensitivity $=(73 / 76) \times 100=96 \cdot 1 \%$. Specificity $=$ $(333 / 340) \times 100=97.9 \%$.

\begin{tabular}{|lcc|}
\hline FPA & \multicolumn{2}{c|}{ Culture } \\
\cline { 2 - 3 } & Positive & Negative \\
\hline Positive & 73 & 7 \\
Negative & 3 & 333 \\
Total & 76 & 340 \\
\hline
\end{tabular}

The serological results of three cases not detected by FPA at admission but detected during post-therapy monitoring are shown in Table 3. Relapse was observed in five cases with positive results of blood culture following therapy.

There is a linear relationship between antibody levels and $\mathrm{mP}$ output of the FPA to a plateau indicating maximum reactivity, somewhere around $250 \mathrm{mP}$, that can be shown by using dilutions of positive sera.

Using a cut-off value of $72 \mathrm{mP}$, FPA detected 80 of 84 sera from suspected brucellosis patients.

Among the 87 sera from probably infected patients without clinical symptoms, the screening test results were PAT (66, $1: 25 ; 20,1: 50 ; 1,1: 100)$, RB (12 positive), BPA (76 positive) and the confirmative tests $\operatorname{TAT}(68,1: 25 ; 17$, $1: 50 ; 1,1: 100)$, CF (19 positive; 1 anticomplementary), CELISA (18 positive) and FPA (19 positive). PAT detected 87 cases and TAT 86; the discrepancy could be due to the occurrence of a non-specific reaction, cross-reacting antigens or false-positive results.

Cross-reactions with antibodies against other micro-organisms do occur in CELISA and FPA, but at a much reduced rate compared with conventional serological tests. No
Table 3. Serological data from three cases of brucellosis receiving treatment, not detected by FPA at admission

Time refers to months after initial isolation of Brucella sp. (species/ biovar in parentheses). $\mathrm{N}$, Negative; + /-, weakly positive; NS, serum not available. See Table 2 for explanation of values shown.

\begin{tabular}{|c|c|c|c|c|}
\hline $\begin{array}{l}\text { Time } \\
\text { (months) }\end{array}$ & TAT & $\mathrm{CF}$ & CELISA & FPA \\
\hline \multicolumn{5}{|c|}{ Case 10 (B. melitensis 1a) } \\
\hline 0 & 400 & 5 & 44 & 63 \\
\hline 3 & 25 & $\mathrm{~N}$ & 19 & 70 \\
\hline 4 & $25+/-$ & $\mathrm{N}$ & 14 & 70 \\
\hline 6 & $\mathrm{~N}$ & $\mathrm{~N}$ & 11 & 68 \\
\hline 12 & $\mathrm{~N}$ & $\mathrm{~N}$ & 10 & 62 \\
\hline \multicolumn{5}{|c|}{ Case 40 (B. abortus 1) } \\
\hline 0 & 800 & 160 & 70 & 66 \\
\hline 3 & 800 & 320 & 51 & 98 \\
\hline 5 & 400 & 160 & 50 & 90 \\
\hline 7 & 200 & 80 & 47 & 97 \\
\hline 12 & 100 & 40 & 36 & 65 \\
\hline \multicolumn{5}{|c|}{ Case 42 (B. abortus 1$)$} \\
\hline 0 & 800 & 80 & 73 & NS \\
\hline 2 & 400 & 80 & 67 & 68 \\
\hline 6 & 200 & 40 & 56 & 124 \\
\hline 9 & 100 & 10 & 57 & 80 \\
\hline 10 & 50 & 5 & 42 & 86 \\
\hline 12 & 100 & 10 & 44 & 78 \\
\hline 13 & 50 & 5 & 43 & 76 \\
\hline
\end{tabular}

evidence of cross-reactions was found for sera from cattle exposed to Yersinia enterocolitica O9 and Escherichia coli O : 157 tested with CELISA and FPA (K. Nielsen, unpublished data).

A large number of serological tests has been used for the diagnosis of human brucellosis, demonstrating the lack of an ideal diagnostic technique and exhibiting limitations with patients in the early phases of the disease, persons exposed

Table 2. Serological results for sera from Brucella sp. culture-positive patients where disagreement between tests was observed

Results considered negative are in bold. Data shown are: TAT, reciprocal of the highest dilution to cause agglutination; CF, reciprocal dilution causing inhibition of haemolysis ( $\mathrm{N}$, negative); CELISA, PI (cut-off is $28 \mathrm{PI}$ ); FPA, $\mathrm{mP}$ (cut-off is $72 \mathrm{mP}$ ). Case 32 was a relapse.

\begin{tabular}{|lrrrrl|}
\hline Case & TAT & CF & CELISA & FPA & \multicolumn{1}{c|}{ Species/biovar } \\
\hline 4 & 200 & 320 & $\mathbf{1 0}$ & 78 & B. melitensis 1 \\
10 & 400 & 5 & 44 & $\mathbf{6 3}$ & B. melitensis 1a \\
28 & $\mathbf{5 0}$ & 10 & 57 & 83 & B. abortus 2 \\
32 & $\mathbf{5 0}$ & 40 & 90 & 219 & B. abortus 1 \\
40 & 800 & 160 & 70 & $\mathbf{6 6}$ & B. abortus 1 \\
52 & 200 & $\mathbf{N}$ & 90 & 218 & B. suis 1a \\
59 & $\mathbf{5 0}$ & $\mathbf{N}$ & 43 & 78 & B. suis 1 \\
\hline
\end{tabular}


professionally and patients who relapse (Ariza et al., 1995; Araj, 1999; Osoba et al., 2001). A marked characteristic of brucellosis is its tendency to produce relapses once treatment is concluded (Solera et al., 1998). The clinical symptoms are often misleading and the disease may present hepatosplenic, osteoarticular, gastrointestinal, neurological and/or cardiovascular complications.

The predominant isotype associated with brucellosis in man appears to be IgG, and is detectable very early in the immune response. Screening tests using acidified antigen preparations, such as BPA and RB, and confirmatory tests such as CF and CELISA have demonstrated an analytical bias in favour of the detection of this isotype. The CELISA has shown $99.7 \%$ specificity and $98.3 \%$ sensitivity and is relatively easy to perform (Lucero et al., 1999).

Using a total of 587 sera in the present study, we investigated a single-step FPA assay as a rapid test for the diagnosis of human brucellosis and as a method to monitor the course of treatment. Tests of 340 sera from healthy people yielded an FPA specificity of $97.9 \%$ when a cut-off value of $72 \mathrm{mP}$ was selected. Seven sera gave false-positive reactions, ranging from 74 to $78 \mathrm{mP}$. Adjusting the cut-off value upwards, specificity may be increased but some sensitivity is lost.

With 76 sera from Brucella-infected patients, the FPA gave a positive result for 73 sera with a sensitivity value of $96 \cdot 1 \%$ using the $72 \mathrm{mP}$ cut-off value. The three false-negative sera gave FPA results ranging from 63 to $68 \mathrm{mP}$. Case 10 was followed up over a 12-month period; however, the FPA titre did not become positive at any time and, after 3 months, all tests were negative. Cases 40 and 42 were FPA-positive 3 months after admission and titres declined slowly over a 12month period (Table 3 ). Forty-four patients from this group were monitored for 2-34 months after initial diagnosis by clinical and laboratory studies including serological tests. In nearly all cases, agreement between CELISA and FPA was good (data not shown). In the same group of patients, FPA and CELISA detected the five cases of relapse (one $B$. melitensis, one B. abortus, three B. suis).

Since a negative blood culture does not exclude the presence of the infection (Yagupsky, 1999) and, at present, there are no definitive criteria to establish that brucellosis has been cured, this study included patients with negative blood culture but clinical symptoms, positive conventional serological tests and CELISA, those most likely infected or who had been exposed to the organism. Of 84 sera monitored from these suspected brucellosis patients, 80 were detected by FPA; the remaining four were patients with declining serological titres.

Of the 87 sera from patients probably infected with Brucella sp., 15 were detected by both FPA and CELISA while three were positive only by CELISA and four only by FPA. Among the latter four patients, one was a meat-packing-plant worker, one a rural worker, one had previous brucellosis history and one was a blood donor.

The discrepancies involve low-titre sera, particularly where titres were declining. In some areas where brucellosis is endemic, there is a high prevalence of antibodies in the healthy population, making individual clinical histories important for correct interpretation of serological test results. By adjusting the cut-off value, CELISA and FPA allow decisions to be made regarding the particular situation depending on requirements of specificity and sensitivity.

An interesting question is whether only immunoglobulins and not other serum components bind to the labelled substrate, but CELISA and FPA are based on the same antigen (OPS). We have evidence that the reaction in CELISA is based on antibody activity and not on other serum proteins, and the high correlation between positive FPA values and cultural evidence of infection as well as other serological evidence indicates a specific antibody response.

The FPA method can be run with a serum sample of $40 \mu \mathrm{l}$, takes about $5 \mathrm{~min}$ to complete and has been demonstrated to be an accurate test for the detection of antibodies to $B$. abortus, B. melitensis and B. suis. Because of the ease of the procedure, it could be adapted for clinical laboratories and blood banks. The test is relatively inexpensive, requiring only a simple buffer, labelled antigen, a reusable glass tube and the equipment required to measure fluorescence polarization, which costs approximately the same as a photometer for enzyme immunoassay. Similar to other primary binding assays and unlike the conventional tests, data are obtained electronically, eliminating subjectivity and providing rapid analysis, a permanent record and easy data dispersal.

\section{ACKNOWLEDGEMENTS}

We are grateful to Dra Ana M. Vigliocco and Romina Ramondino at the Comisión Nacional de Energía Atómica, Buenos Aires, Argentina, for their help.

\section{REFERENCES}

Alton, G. G., Jones, L. M., Angus, R. D. \& Verger, J. M. (1988). Serological methods. In Techniques for the Brucellosis Laboratory, pp. 63-134. Paris: Institut National de la Recherche Agronomique.

Angus, R. D. \& Barton, C. E. (1984). The production and evaluation of a buffered plate antigen for use in a presumptive test for brucellosis. Dev Biol Stand 56, 349-356.

Araj, G. F. (1999). Human brucellosis: a classical infectious disease with persistent diagnostic challenges. Clin Lab Sci 12, 207-212.

Ariza, J., Corredoira, J., Pallares, R., Viladrich, P. F., Rufi, G., Pujol, M. \& Gudiol, F. (1995). Characteristics of and risk factors for relapse of brucellosis in humans. Clin Infect Dis 20, 1241-1249.

Centro Panamericano de Zoonosis (1981). Brucelosis: prueba de fijación de complemento. Nota Técnica no. 24. Buenos Aires: CEPANZO (in Spanish).

Corbel, M. J. \& Brinley-Morgan, W. J. (1984). Genus Brucella Meyer and Shaw 1920, 173 ${ }^{\text {AL }}$. In Bergey's Manual of Systematic Bacteriology, vol. 1, pp. 377-388. Edited by N. R. Krieg \& J. G. Holt. Baltimore: Williams \& Wilkins.

Corbel, M. J., Thomas, E. L. \& Garcia-Carrillo, C. (1984). Taxonomic studies on some atypical strains of Brucella suis. Br Vet J 140, 34-43.

Garcia-Carrillo, C. \& Lucero, N. E. (1993). Diagnóstico bacteriológico. In Brucelosis Bovina, pp. 97-98. Edited by A. I. De Diego. Buenos Aires: Hemisferio Sur (in Spanish). 
Lucero, N. E. \& Bolpe, J. E. (1998). Buffered plate antigen test as a screening test for diagnosis of human brucellosis. J Clin Microbiol 36, $1425-1427$.

Lucero, N. E., Foglia, L., Ayala, S. M., Gall, D. \& Nielsen, K. (1999). Competitive enzyme immunoassay for diagnosis of human brucellosis. J Clin Microbiol 37, 3245-3248.

Metz, C. E. (1978). Basic principles of ROC analysis. Semin Nucl Med 8, 283-298.

Nielsen, K. H., Kelly, L., Gall, D., Nicoletti, P. \& Kelly, W. (1995). Improved competitive enzyme immunoassay for the diagnosis of bovine brucellosis. Vet Immunol Immunopathol 46, 285-291.

Nielsen, K. H., Gall, D., Kelly, W., Vigliocco, A., Henning, D. \& Garcia, M. (1996). Immunoassay Development: Application to Enzyme Immunoassay for the Diagnosis of Brucellosis. Nepean, Ontario: Agriculture and Agri-Food Canada.
Nielsen, K., Lin, M., Gall, D. \& Jolley, M. (2000). Fluorescence polarization immunoassay: detection of antibody to Brucella abortus. Methods 22, 71-76.

Osoba, A. O., Balkhy, H., Memish, Z., Khan, M. Y., Al-Thagafi, A., Al Shareef, B., Al Mowallad, A. \& Oni, G. A. (2001). Diagnostic value of Brucella ELISA IgG and IgM in bacteremic and non-bacteremic patients with brucellosis. J Chemother 13 (Suppl. 1), 54-59.

Schoonjans, F., Zalata, A., Depuydt, C. E. \& Comhaire, F. H. (1995). MedCalc: a new computer program for medical statistics. Comput Methods Programs Biomed 48, 257-262.

Solera, J., Martinez-Alfaro, E., Espinoza, A., Castillejos, M., L., Geijo, P. \& Rodriguez-Zapata, M. (1998). Multivariate model for predicting relapse in human brucellosis. J Infect 36, 85-92.

Yagupsky, P. (1999). Detection of brucellae in blood cultures. J Clin Microbiol 37, 3437-3442. 